\title{
Wireless Video Performance Through BLAST Testbed
}

\author{
Haitao Zheng and Dragan Samardzija \\ Wireless Communications Research Department \\ 791 Holmdel-Keyport Rd. Holmdel, NJ 07733 \\ Email: haitaoz,dragan@lucent.com
}

\begin{abstract}
Multiple antenna system has been shown to provide high capacity wireless communications. We have built a narrowband wireless BLAST testbed with multiple transmit and receive antennas. To validate the effectiveness of the testbed and BLAST technique, we transmit a $\mathrm{H} .263$ video at a rate of $230 \mathrm{kbps}$. The video performance under different channel conditions and error handling options are discussed.
\end{abstract}

\section{INTRODUCTION}

The Bell Labs Layered Space-Time (BLAST) or MIMo [1] architecture utilizes multi-element antenna arrays at both transmitter and receiver to provide high capacity wireless communications in a rich scattering environment. It has been shown that the theoretical capacity increases linearly as the number of antennas is increased. VBLAST[2] is a simplified version where channel coding is applied to individual sub-layer, each corresponding to the data stream transmitted by a single antenna. DBLAST applies coding not only across the time, but also across the antennas (sub-layers), and implies higher complexity.

We have built a narrowband wireless testbed based on VBLAST, which is used for verifications and performance evaluations of different algorithms related to the BLAST wireless communication architecture. 'To illustrate the high capacity gain provided by BLAST, we perform transmission of $\mathrm{H} .263$ video coded at $230 \mathrm{kbps}$ over the VBLAST testbed and the performances under different channel designs are studied.

\section{NARROWBAND VBLAST TESTBED}

Let us now describe the hardware components of the narrowband VBLAST wireless testbed. Radio frequency (RF) front end of the testbed consists of an antenna array, and the corresponding array of analog RF transmitters and receivers. In this particular experiment we used up to eight transmit and eight receive antennas. The carrier is at $1.95 \mathrm{GHz}$ and the signal bandwidth is limited to $30 \mathrm{KHz}$. The baseband digital signal processing is executed using a DSP multiprocessor system. The maximum sampling rate, per a baseband channel, is $100 \mathrm{KHz}$. We use the QPSK modulation format, transmitting at $25 \mathrm{Ksym} / \mathrm{sec}$, per subuser (i.e., per antenna). Further, the symbols are organized as in Figure 1. Symbols 1 to 16 are used for synchronization, i.e., frame and symbol timing recovery. Note this part of the frame is identical for all the subusers. Symbols 17 to 32 compose a training sequence, which is used for estimation of the channel response. Between the subusers, the sequences are mutually orthogonal and with equal transmission power. Symbols 33 to 232 are information-bearing symbols. 
Considering the QPSK format, 400 bits are transmitted per frame, per transmit antenna.

Predefined, non information bearing symbols

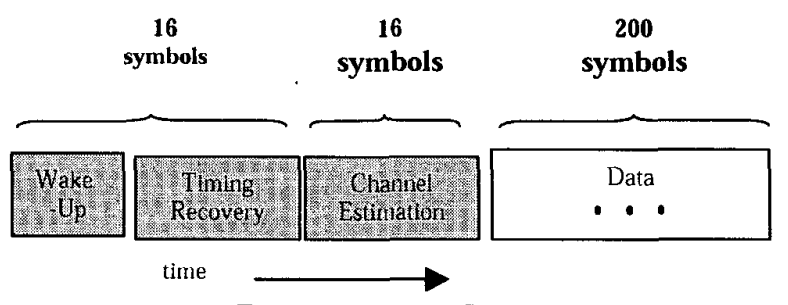

Figure 1. Frame Structure

The receiver consists of the following baseband digital signal processing blocks, more details can be found in [3].

A. Detection of the frame start.

F. Symbol timing recovery.

C. Mitigation of hardware induced intersymbol interference (ISI).

I). Estimation of the channel response.

E.. The VBLAST Algorithm.

In this experiment, each frame consists of 232 QPSK symbols, where 32 symbols are dedicated to synchronization and training. Therefore, 200 QPSK symbols are transmitted through each antenna. To achieve better coding efficiency, one single convolutional code is applied to all the subusers. We employ rate $1 / 2$ and $1 / 3$ convolutional codes of constraint length 8 . In addition, a rate $2 / 3$ code is obtained by puncturing the output of the rate $1 / 3$ code. Using $8 \times 8$ system as a example, by multiplexing the coded bits into 8 sub-users, an interleaving of depth 8 is achieved naturally. At the receiver, the VBLAST algorithm is applied to extract the soft input, which is forwarded to the channel decoder. The data rate per transmit antenna when there is no coding can be computed as $200 * 2 /(9.28 \mathrm{e}-3)=43.10 \mathrm{kbps}$. The frame structures for different coding rates are illustrated in Table 1.

Table 1. Frame Structure

$9.28 \mathrm{~ms}$ frame, 200 QPSK symbols per frame per Tx antenna

\begin{tabular}{|l|l|l|l|l|}
\hline \multirow{2}{*}{$\begin{array}{l}\text { Coding } \\
\text { Rate }\end{array}$} & \multicolumn{2}{|c|}{$6 \mathrm{Tx}$} & \multicolumn{2}{c|}{$8 \mathrm{Tx}$} \\
\cline { 2 - 5 } & Info & throughput & Info & Throughput \\
\hline 1 & 2400 & $258.6 \mathrm{kbps}$ & 3200 & $345 \mathrm{kbps}$ \\
\hline $2 / 3$ & 1600 & $172.4 \mathrm{kbps}$ & 2125 & $230 \mathrm{kbps}$ \\
\hline $1 / 2$ & 1200 & $129.3 \mathrm{kbps}$ & 1600 & $172.4 \mathrm{kbps}$ \\
\hline $1 / 3$ & 800 & $86.14 \mathrm{kbps}$ & 1058 & $115 \mathrm{kbps}$ \\
\hline
\end{tabular}

\section{3. $\quad$ SYSTEM STRUCTURE}

We are interested to validate VBLAST testbed performance by transmitting video data. The video quality is presented in terms of the Peak Signal-to-Noise Ratio (PSNR) of the video. The system structure is shown in Figure 2. We choose an 
H.263+ coded video sequence, with a bit rate of $230 \mathrm{kbps}$ at $15 \mathrm{fps}$. The following error-resilience features were implemented: 1 ) inserting one intra frame every five frames, 2) insert sync word in each GOB(slice). For more information about the H.263+ video coding technique, see [4].

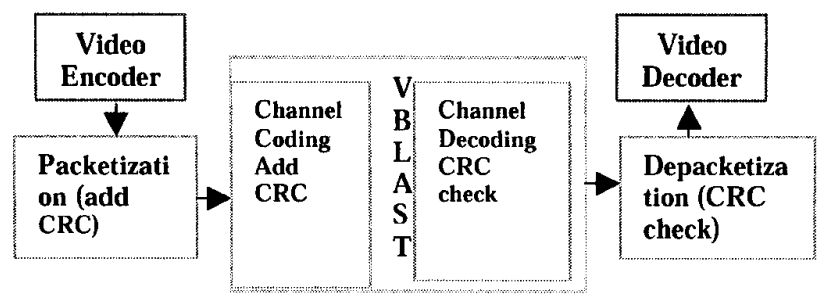

Figure 2. System Architecture

The video stream is packetized through the detection of GOB synchronization word. In another word, each GOB corresponds to one application packet, and the resulted packets are of different lengths. Each packet is accompanied by a 16-bit $\mathrm{CRC}$ check for content validation. Channel errors usually partially corrupt a packet. If the protocol discards a packet containing only a small part of corrupted data, it also throws out error-free data within the packet. Indeed, the media decoder can detect and tolerate a certain amount of channel errors. To support this feature, it would be possible to still forward the corrupted packet to the video decoder and let the video decoder to detect the errors. Therefore, when the packet $\mathrm{CRC}$ fails, we consider the following two options for comparison:

I. Discard the packet

II. Forward the packet to video decoder

On the other hand, each physical layer frame is accompanied by a 16-bit CRC check. At the receiver, nearly all the errors can be detected. This indeed provides an accurate error indication. However, in the conventional system design, the physical layer does not communicate with the application layer. And it might simply discard the frame. For video/audio, this could generate additional errors. Therefore, we have proposed to forward the frame error indication to the application layer[5]. One example would be replacing the corrupted physical layer frames as all $1 \mathrm{~s}$, which can be recognized by the media decoder as an invalid codeword and thus invokes error concealment to reduce or even eliminate the error effect. When video decoder is effective in terms of error detection, physical and network layer can simply forward the corrupted frames/packets to the video decoder for flexible error control. In this experiment, when CRC detects channel error, we compare three options in terms of error handling in physical layer:

A. Discard the frame

B. Forward the frame to video depacketization.

C. Replace the frame as all ones

It should be noted that by employing option I in packet level error handling, i.e. discarding the packet, the performance remains the same for option $A$ to $C$. Therefore, we simply compare the following four options in terms of packet level and physical layer level error handling techniques: I, II+A, II+B, II+C. 


\section{EXPERIMENTAL RESULTS}

We use $(M, N)$ to represent a BLAST system with $M$ transmit antennas and $N$ receive antennas. In this paper, we evaluated both $(6,8)$ and $(8,8)$ VBLAST in terms of link adaptation through coding rate selection, and antenna selection. First we examine the effectiveness of the channel coding by looking at the error traces before and after channel coding. Figure 2 left illustrates the received frame error trace assuming no channel coding, where $30 \%$ frame error rate is obtained. The $y$-axis also shows the number of bit errors within the corrupted frames. The raw frame errors are in fact bursty. The performance with rate $2 / 3$ channel coding is shown in Figure 2 right. The frame error rate is reduced to $3 \%$ and the bursty error effect disappears.
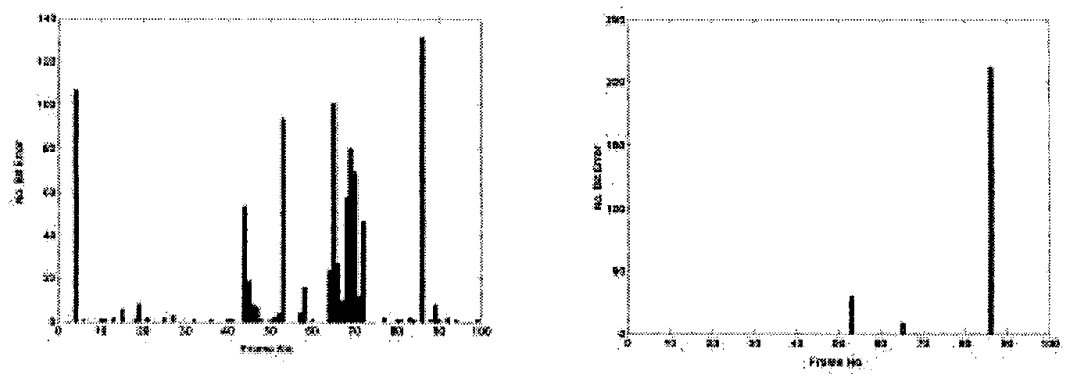

Figure 2. Frame Errors before (left) and after(right) rate 2/3 channel coding.

To find the appropriate coding rate, Figure 3 illustrates the throughput performance for a $(6,8)$ system at coding rates of $2 / 3$ and $1 / 2$. The throughput is computed as $R(1-F E R)$ where $R$ represents the number of information bits carried per second and FER is the frame error rate. It is not surprise to find that the optimal coding rate depends on the SNR. Therefore, the system should employ link adaptation to maximize the link throughput.

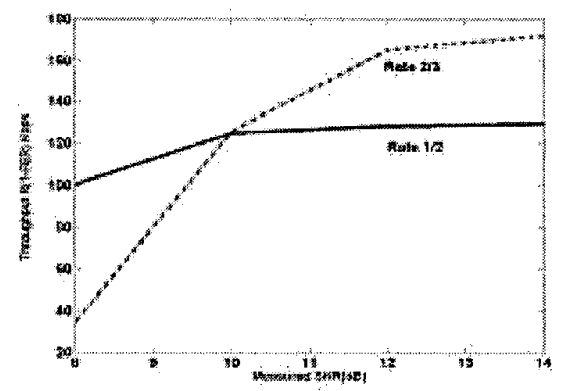

Figure 3. Throughput Performances for $(6,8)$ BLAST with rate $2 / 3$ and $1 / 2$ convolutional channel

The concept of BLAST is to utilize both receive and transmit diversity to boost data rate, but to choose the number of transmit and receive antenna elements 
depends on both performance and complexity/cost. For VBLAST, where each transmit antenna yields spatial interference to the others, using all the transmit antennas might not yield the best performance. Therefore, for a given number of receive antennas, we need to select an optimal number of transmit antennas. On the other hand, it is well known that adding receive antennas always improve the system performance. So that the best number of receive antennas only depends on the hardware complexity and cost. In this paper, we fix the number of receive antenna elements to 8 . In Figure 3 right, we compare the throughput performance of a $(6,8)$ system to a $(8,8)$ system. The link adaptation by choosing coding rates is reflected in the curve. It should be pointed out that the total power constraint is fixed during the comparison. In another word, the amount of power transmitted from each antenna would be larger for $(6,8)$ compared to that of $(8,8)$. The results show that the selection depends on the SNR value, but for a typical SNR range of $5-12 \mathrm{~dB},(6,8)$ VBLAST is superior to $(8,8)$.

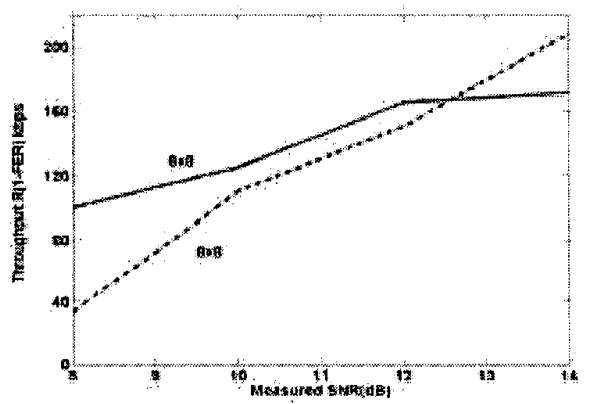

Figure 4. Throughput Performances for $(6,8)$ and $(8,8)$ antenna configurations.

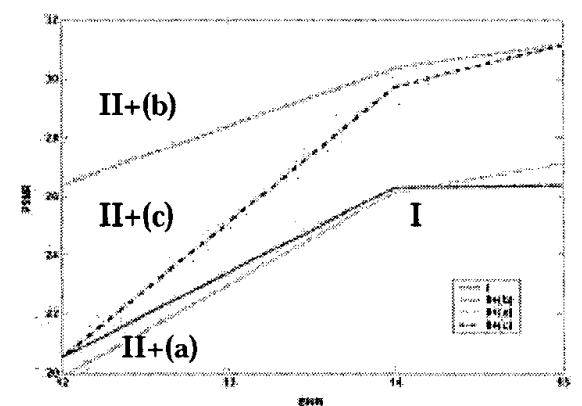

Figure 5. Video Performance

From Figure 3 and 4, we observe that a data rate of $230 \mathrm{kbps}$ can be achieved when SNR is higher than $14 \mathrm{~dB}$. It should be pointed out that for lower SNRs we could reduce the video-coding rate to avoid packet loss and error. However, since this paper is focused on demonstrating the error handling techniques when the channel variation is difficult to predict, we choose to simulate within 12 to $15 \mathrm{~dB}$ SNR range. We use the error traces generated from the real-time testbed results to simulate the video performance. The error traces reflects $1.5 \%-16 \%$ FER. The PSNR performances are obtained by averaging the results from 20 experiments. 
Figure 5 depicts that forwarding all the physical layer data to the application layer is the best solution for this type of channel. This is mainly due to that the number of error bits within corrupted frames are fairly small so that the video decoder can easily detect and conceal the channel errors. For II+C option, we see graduate performance improvement as SNR increases, but still it achieves as much as $4 \mathrm{~dB}$ FSNR improvement compared to the other two options. Therefore, it is concluded that the protocol architecture design greatly impacts the system performance.

\section{CONCLUSION}

In this paper, we simulate wireless video transmissions using a narrowband VBLAST testbed with multiple transmit and receive antenna elements. We define the VBLAST testbed architecture by choosing the coding rate, link adaptation region and the number of transmit and receiver antenna elements. It is observed that simple convolutional codes can significantly reduce the frame error and link adaptation can be achieved by varying the coding rates. On the other hand, for a fixed 8 receive antenna elements, activating 6 transmit antennas rather than 8 yields higher throughput when SNR is lower than $13 \mathrm{~dB}$. Real over the air error traces are extracted from the testbed and applied to video transmission. We discussed the error handling techniques at both packet level and physical frame level. Various options are compared by evaluating the received video quality. We found that for the environments that the testbed experienced, the number of bit error is small within each corrupted frame. Under this condition, forwarding all the frames and packets to the application layer/video decoder achieves the best video performance. For wireless communications, the physical layer is usually the performance bottleneck and difficult to improve due to large and unpredictable channel variation and multipath fading. For specific applications like multimedia data with error resilience, one can design the layer 2 and 3(MAC, network) wisely to achieve better error recovery at the application layer. As shown in the video performance, this consideration can dramatically improve the overall performance without putting huge complexity in improving physical layer performance.

\section{REFERENCE}

[1] G. J. Foschini, "Layered Space-Time Architecture for Wireless Communication in a Fading Environment When Using Multiple Antennas", Bell Labs Technical Journal, Vol. 1, No. 2, Autumn 1996, pp 41-59.

[2] G. D. Golden, G. J. Foschini, R. A. Valenzuela, P. W. Wolniansky, "Detection Algorithm and Initial Laboratory Results using the V-BLAST Space-Time Communication Architecture", Elec. Letters, Vol. 35, No.1, Jan.1999, pp. 14-15.

[3] H. Zheng and D. Samardzija, "On the Performance of H.263 Video over Multiple Antenna Systems", CISS 01, March 2001.

[4] ITU Recommendation H.263 Version 2, Video Coding for Low Bitrate Communication, Jan. 1998.

[5] H. Zheng and J. Boyce, "Packet Coding Schemes for MPEG Video over Internet and Wireless Networks", IEEE WCNC, Sept. 2000, Chicago, IL. 